\title{
Microfluidic platform for studying biofilm formation of oil-degrading bacteria
}

\author{
Saeed Rismani Yazdi, Carlos Escobedo \\ Department of Chemical Engineering \\ Queen's University \\ Kingston, Ontario, Canada \\ carlos.escobedo@queensu.ca
}

\author{
Corey A. Stevens, Peter L. Davies \\ Department of Biomedical and Molecular Sciences \\ Queen's University \\ Kingston, Ontario, Canada
}

\begin{abstract}
Crude oil spill is a significant threat to marine wildlife and human health. Understanding the interaction of oil-degrading bacteria at aqueous-oil interfaces at submillimeter scale is crucial to biodegradation and bioremediation. Here we present a microfluidic system that allows real-time and parallel observation of bacteria interaction with crude oil during biofilm formation and degradation of the oil. A simple and robust microfluidic platform is designed, fabricated and tested. Stable and controllable oil-bacterial solution interfaces are formed. The theory of the capillary valves used in the device and the microfabrication steps are presented. Experimental results show the accumulation of Marinobacter hydrocarbonoclasticus -an oil degrading bacteria- at the bacterial solution interface with paraffin oil. After $\sim 3 \mathrm{hr}$ of incubation, bacteria start to form microcolony at the oil interface. Results show that presented microfluidic device can be successfully used for research on bacteria-oil interactions and other applications requiring an interface formed by two immiscible liquids.
\end{abstract}

Keywords- microfluidics; oil-degrading bacteria; biofilm; interfaces; capillary valve

\section{INTRODUCTION}

Crude oil spill is a significant threat to marine wildlife and human health. Each year, 700 million tons of crude oil is introduced to aquatic ecosystem through human related activities and by natural seepage from reservoirs [1], [2]. This, therefore, has motivated more progress in marine oil spill remediation studies [3], [4]. Nature has evolved an effective way to rectify the problem using indigenous microorganisms to clean up the oil contaminants [5]. Marinobacter hydrocarbonoclasticus (MH) is an aquatic bacterium with the ability to form biofilms at the interface between water and oil [6]. MH uses this ability to increase the bioavailability of hydrocarbons, which serve as its main energy source, and further to degrade the oil. Biofilms have been previously studied using macroscopic approaches [7]. Analysis at microscales, however, can provide key information on the dynamics of biofilm formation and disruption [8], [9]. A central challenge for studying $\mathrm{MH}$ is to form a stable and observable oil-bacterial solution interface that mimics those found in natural settings.

Over the last decade, the lab-on-a-chip technology and in particular microfluidics has been maturing, leading to tremendous applications in life science and engineering [10]. In general, microfluidics can help to overcome challenges and limitations of convectional assays, for example in biology and chemistry, and also allow development of approaches that can lead to better understanding of natural or engineered phenomenon at submillimeter scale [11]. Microfluidics offers unique advantages such as low sample and reagent volumes ( $\mu \mathrm{L}$ to $\mathrm{pL}$ ), precise control of spatial and temporal factors with high sensitivity, low-cost, with simplified fabrication and operation steps [10], [12]. Moreover, the use of biocompatible and optically transparent materials such as Polydimethylsiloxane (PDMS) facilitates the culture of biological samples and image acquisition using conventional microscopy [13]. These advantages make microfluidics an ideal tool for studying the interaction of Marinobacter hydrocarbonoclasticus with oil.

Here we present a simple and versatile microfluidic device for studying bacteria with oil degrading ability as a model for marine bacteria interaction with crude oil during biofilm formation. Using capillary valves incorporated in the microfluidic device, the oil-bacterial solution interface is realized. The principle behind the capillary valves used in the microfluidic device is presented. Use of such microfluidic device with passive valves is advantageous because it does not require complex setup, thus increasing the simplicity of the fabrication and liquid handling in the device. The design of the device allows for experimental parallelization with different conditions at a time to investigate, for instance, the effects of two different oils or promoting/inhibitory reagents on the bacteria interaction with the oil.

\section{RESULTS AND DISCUSSION}

\section{A. Microfluidic device}

Fig. 1 shows the schematic representation of the microfluidic device for studying bacteria interaction with oil. 
The device consists of three parallel channels that allow the formation of immiscible liquid-liquid interfaces. The middle and the two adjacent channels are interconnected through 100 $\mu \mathrm{m}$ gaps between 20 trapezoidal posts located in series (Fig. 1). All channels have $40 \mu \mathrm{m}$ height. The abrupt angle of the posts geometry increases the physical contact angle between the solid-liquid interface, resulting in a capillary valve action [14], which consequently retains the meniscus between the microposts and prevents the liquid in the middle channel from flowing into the side channels. The middle channel can be filled with oil and side channels with bacteria solution to bring the two liquids into contact (Fig. 1). Fig. 2 shows the PDMS microfluidic device fabricated using photolithography and soft lithography techniques as described in the method section [15].

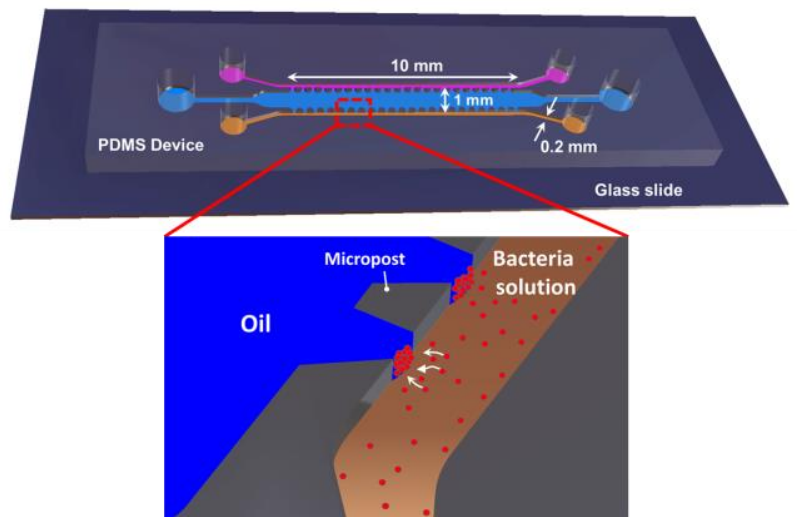

Figure 1. Architecture of the microfluidic device developed to study interaction of Marinobacter hydrocarbonoclasticus and oil. Liquid-liquid interfaces are achieved at the meniscuses formed as a result of the gaps between the microposts.

\section{B. Capillary valves}

The capillary valves stop the liquid from flowing to a certain region of the microfluidic device using a capillary pressure barrier [14]-[16]. For this particular type of valve, the capillary pressure barrier develops when abrupt enlargement of the channel happens. The valve consists of a region of the channel that is necked down followed by a sharp expansion of the cross-section that locally increases of the static contact angle. In the proposed microfluidic device, the implemented micorposts work as capillary valves (Fig. 2).

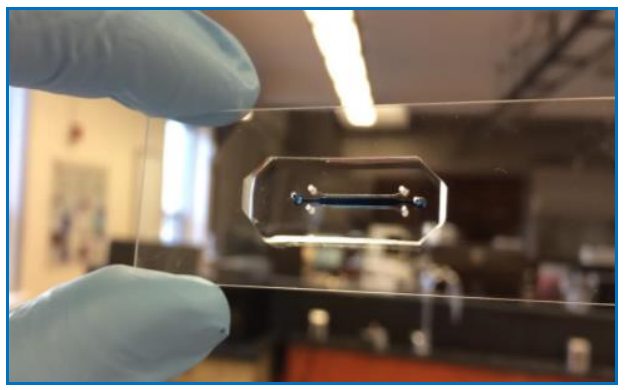

Figure 2. Image of fabricated PDMS microfluidic device. The loaded blue liquid is stopped from flowing to the side channels by the microposts acting as capillary valves.

The capillary valves stop the liquid from flowing to a certain region of the microfluidic device using a capillary pressure barrier [14]-[16]. For this particular type of valve, the capillary pressure barrier develops when abrupt enlargement of the channel happens. The valve consists of a region of the channel that is necked down followed by a sharp expansion of the cross-section that locally increases of the static contact angle. The loaded liquid in the microfluidic device drives by the combination of the external pressure and capillary action in the channel and between microposts (necked regions, Fig. 3a). The abrupt enlargement of the edge of the microposts $\left(145^{\circ}\right)$ prevented the liquid from flooding to the side channel using the pressure barrier (Fig. 3b). This can be explained in terms of changes in the energy for the liquid-solid-air interface system. The total interfacial energy $\left(U_{T}\right)$ of capillary system can be presented by:

$$
\mathrm{U}_{\mathrm{T}}=\mathrm{ASL}_{\mathrm{SL}} \gamma_{\mathrm{SL}}+\mathrm{ASA}_{\mathrm{SA}} \gamma_{\mathrm{SA}}+\mathrm{A}_{\mathrm{LA}} \gamma_{\mathrm{LA}}
$$

where $A_{S L}$ and $\gamma_{S L}, A_{S A}$ and $\gamma_{S A}, A_{L A}$ and $\gamma_{L A}$ are solid-liquid, solid-air, and liquid-air interface areas and surface energies per unit area, respectively [16], [17]. The relation between surface energies of triple interfaces and the contact angle of liquid $\theta_{\mathrm{C}}$ with the surface of microfluidic channel at the meniscus can be expressed by Young's equation:

$$
\gamma_{\mathrm{SA}}=\gamma_{\mathrm{SL}}+\gamma_{\mathrm{LA}} \cos \theta_{\mathrm{C}}
$$

The $U_{T}$ of the system is a function of loaded volume liquid $\left(V_{L}\right)$ into the device as wetted surfaces change by increase of the $V_{L}$.

$$
P=-\frac{d U_{\mathrm{T}}}{d V_{\mathrm{L}}}=\gamma_{\mathrm{LA}}\left(\cos \theta_{c} \frac{d A_{\mathrm{SL}}}{d V_{\mathrm{L}}}-\frac{d A_{\mathrm{LA}}}{d V_{\mathrm{L}}}\right)
$$

When liquid is loaded, it wets the surfaces of the channels which increases the liquid-solid interface and thus the positive pressure moves the liquid forward and fills the channel. In order to stop the liquid from going to the side channels, pressure as driving force should be controlled. This can be achieved by geometry change of the valve. The abrupt geometry change of valve results in an increase of liquid-air interface more than solid-liquid interface at the end of the tapered edge, which leads to opposite sign of the pressure at the meniscus. The negative pressure is a repulsive force and thus stops the filling front at the edge of the valve. When the liquid reaches the sharp edge of the valve, the filling front stops and meniscus remains at the edge unless higher pressure is applied to the liquid which breaks the pressure barrier and liquid flows to the side channels. The Young-Laplace equation expresses the inverse relation of pressure across the liquid-air interface and the radius of meniscus. According to the equation 4 , the pressure at the liquid-air interface increases when the meniscus radius (r) gets smaller or in other words when meniscus becomes more convex [16], [18].

$$
\Delta p=p-p_{n}=\frac{2 \gamma_{\mathrm{LA}}}{\mathrm{r}}
$$

Depending on the difference between internal pressure $(p)$ of the liquid and normal pressure in the side channel $\left(p_{n}\right)$, the liquid-air interface bulges more or less. For a given volume, if further illiquid is gradually loaded to the channel, the liquid continues to move and meniscus shape changes from concave to flat and then to convex (Fig. 3). If the volume of the liquid decreases, the meniscus shape changes back to the concave. 
This is a reversible action until the meniscus is pinned and valve is not open. Here we assume that all the surfaces in the microchannel for PDMS and glass are homogenous and have same surface properties (e.i. hydrophobic).

a

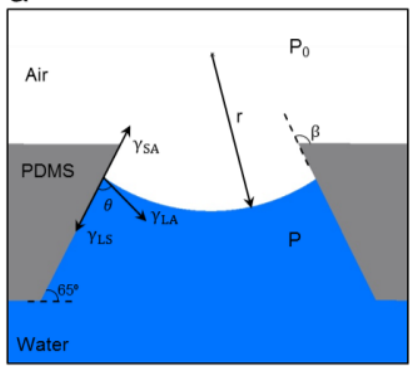

Figure 3. Principle of the capillary valves. (a) Filling front of the liquid while reaching the sharp edge of the valve and (b) the pinned meniscus due to the pressure barrier from the valve.

\section{Formation of liquid-liquid interface}

To test the functionality of the device, first the middle channel was filled with the oil (colored in green in Fig. 4) and after the oil filling front between the microposts was stopped by the valves, the side channels were filled with the water (colored in pink in Fig. 4). Fig. 4 shows the well-defined oilwater interfaces formed between microposts. Similar interfaces achived for all the 20 gaps between microposts. By modulating the volume of liquid loaded into the middle channel, meniscus shape can be altered and concave, flat, and convex meniscus can be achieved (Fig. 5).

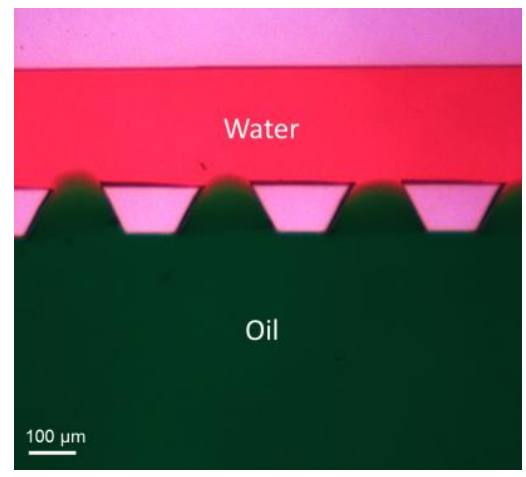

Figure 4. Stable and well-defined oil-water interfaces formed between microposts. Oil is colored in green and water in pink.

The effectiveness of the valves to pin and maintain the meniscus during the experiments were tested and results showed that oil-bacterial solution interfaces were stable over the course of the experiment for $>5 \mathrm{hr}$. Using this approach, it is possible to bring two immiscible liquids into contact and form a liquid-liquid interface, such as oil-water, between the microposts.
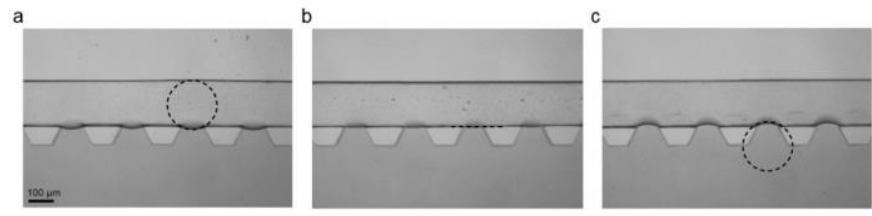

Figure 5. Zoomed view of the middle channel filled with water. By varying the volume of liquid loaded to the channel, the meniscus shape can be controlled to be a) convex, b) flat, and c) concave. Side channel was left empty. The dashed line and circles show the direction of the meniscus.

\section{Bacteria interaction with oil}

In order to study biofilms formation at the oil-water interface, the middle channel was loaded with metabolisable hydrocarbon and the side channels were filled with highly concentrated salt solution containing FITC-labeled $M$. hydrocarbonoclasticus. The experimental results demonstrate observable oil-bacterial solution interfaces between all microposts and substantial bacteria accumulation at the oil interfaces (Fig. 6). Due to the migration of the bacteria towards the oil and their accumulation there, higher number of bacteria were found close to the oil interface (Fig. 6).
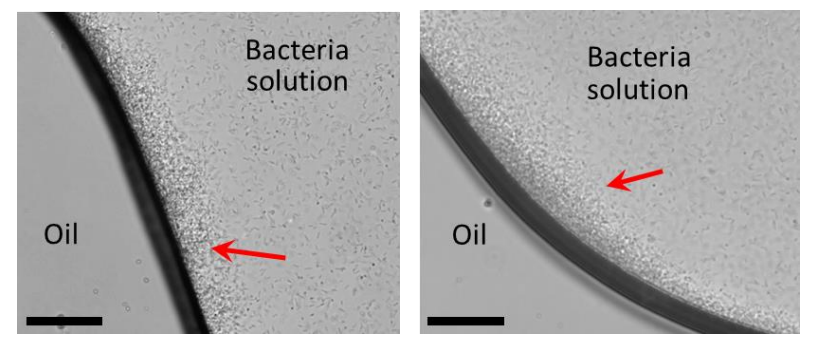

Figure 6. Accumulation of Marinobacter hydrocarbonoclasticus at the bacterial solution interface with paraffin oil. Scale bars, $50 \mu \mathrm{m}$. Red arrows show the area with bacteria accumulated at the oil interface.

Fig. 7 shows the images of the oil-bacteria interface at time zero (Fig. 7-left) and at time $\sim 3 \mathrm{hr}$ (Fig. 7-right). The accumolation of the bacteria and formation of the microcolony in the microfluidc device was imaged over time. After 3 hours of incubation, marinobacter bacteria started to form biofilm/microcolony at the interface with the parafin oil. Results show the successful formation of stable and observable oil-bacterial solution interfaces that can mimics those found in natural settings. The posibility to have 20 interfaces at each side of the middle channel improves the number of data points and statistical analysis to support the observed phenomenons.
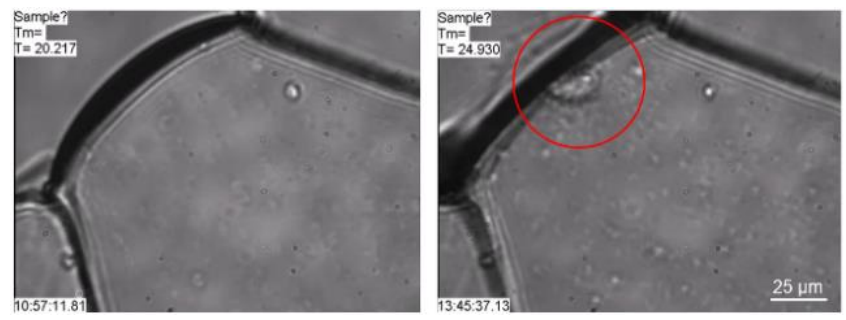

Figure 7. Formation of a biofilm/microcolony between marinobacter and parafin oil after $\sim 3$ hours of incubation in $20^{\circ} \mathrm{c}$ in the microfluidic device. The microcolony is shown by red circle.

\section{CONCLUSION}

In summary, a simple and robust microfluidic device allowing the formation of oil-water interfaces was presented and used to study interaction of Marinobacter hydrocarbonoclasticus - an oil degrading bacteria - with parafine oil. The device utilized capillary valves formed by the sharp edges of 20 microposts that connect the two channels 
together and allowed for controllable and observable interfaces between oil and bacterial solution. The proposed microfluidic device enabled experimental parallelization with different conditions at a time to investigate, for instance, the effects of two different oils or promoting/inhibitory reagents on the biofilm formation. In order to study biofilm formation at the oil-water interface, the middle channel can be loaded with a highly concentrated salt solution containing Marinobacter hydrocarbonoclasticus, while the side channels can be filled with a degradable and non-degradable oil such as Hexadecane and 1-Octanol, respectively. This versatile platform has great potential not only for studying oleolytic biofilms, but also for applications that require interfaces formed by two immiscible liquids.

\section{MATERIALS AND METHODS}

\section{A. Fabrication of microfluidic device}

The microfluidic device was fabricated using photolithography and soft lithography techniques [19]. Microfluidic networks were designed in CATIA (Dassault Systems, V19). For maximum process reliability, the silicon wafer was cleaned with acetone and isopropanol, and then rinsed with water for 5 minutes. The wafer was then dried on a $100{ }^{\circ} \mathrm{C}$ hotplate. A $40 \mu \mathrm{m}$ layer of SU-8 50 (MicroChem, USA) was spin-coated on a silicon wafer and selectively exposed to ultraviolet light using a maskless photolithography system (Intelligent Micro Patterning Co., USA). SU-8 crosslinking was obtained at near UV (350-400 nm) radiations for 3 $\mathrm{s}$ with $\sim 35 \mathrm{~mW} / \mathrm{cm}^{2}$ exposure energy. The unexposed photoresist was developed in the SU-8 developer (MicroChem, USA) for 20 minutes. The exposure and development time were optimized to achieve sharp edges for the microposts. The developed SU-8 mold was silanized with (tridecafluoro-1,1,2,2-tetrahydrooctyl)-1-trichlorosilane (Sigma-Aldrich, Switzerland) to reduce adhesion between the SU-8 mold and PDMS. PDMS precursor and crosslinking agent (Sylgard 184 Silicone Elastomer, Dow Corning Corp., USA) were mixed at a ratio of 10 to 1 , based on weight, and degassed in a vacuum chamber for $30 \mathrm{~min}$. Then, the PDMS was poured on the mold, degassed, and cured at $80^{\circ} \mathrm{C}$ for at least 1 hour. The cured PDMS layer was peeled off from the SU-8 mold and a hole puncher (1 $\mathrm{mm}$ in diameter) was used to punch the inlet and outlet ports. A microscope slide (Microscopes America, Inc., USA) was bonded to the PDMS layer using a plasma cleaner (Harrick Plasma Inc., USA) to complete the microfluidic device.

\section{B. Experimental procedure and microscopy}

Microchannel inlets were connected to syringes containing the oil and bacteria solutions using silicon tubes (TYGON R3607, Ismatec) and 21-gauge 90-degree-bent needle (APM Technica). Marinobacter hydrocarbonoclasticus culture of 2 days old was used for the experiments. Prior to the experiments, bacteria were centrifuged for $10 \mathrm{~min}$ at $2000 \mathrm{rpm}$ and then resuspended in $200 \mu \mathrm{l}$ of the culture medium. After loading the channels, microfluidic device was placed into the incubator stage of an inverted microscope for temperature and humidity control. An inverted microscope (Olympus IX83,
Germany) equipped with a CMOS camera (Zyla-4.2-CL10, ANDOR, Ireland) was used to capture 10x (numerical aperture $(\mathrm{NA})=0.13$, UPLanFL $\mathrm{N}$ ) and 40x (NA 0.6, LUCPlanFL N) magnification bright-field image sequences of bacteria interacting with the oil in the microfluidic device. Freely available image processing software ImageJ (US National Institutes of Health) [20] was used to perform image analysis.

\section{ACKNOWLEDGMENT}

This work was supported by Natural Sciences and Engineering Research Council of Canada (NSERC) Discovery Grants to CE and PLD, and a Canada Foundation for Innovation Leaders Opportunity Fund program.

\section{REFERENCES}

[1] S. Kleindienst, J. H. Paul, and S. B. Joye, "Using dispersants after oil spills: impacts on the composition and activity of microbial communities.," Nat. Rev. Microbiol., vol. 13, no. 6, pp. 388-396, 2015.

[2] I. R. MacDonald, I. Leifer, R. Sassen, P. Stine, R. Mitchell, and N. Guinasso, "Transfer of hydrocarbons from natural seeps to the water column and atmosphere," Geofluids, vol. 2, no. 2, pp. 95-107, 2002.

[3] H. P. Bacosa, Z. Liu, and D. L. Erdner, "Natural sunlight shapes crude oil-degrading bacterial communities in northern Gulf of Mexico surface waters," Front. Microbiol., vol. 6, no. DEC, 2015.

[4] T. J. McGenity, B. D. Folwell, B. a McKew, and G. O. Sanni, "Marine crude-oil biodegradation: a central role for interspecies interactions," Aquat. Biosyst., vol. 8, no. 1, p. 10, 2012.

[5] J. G. Leahy and R. R. Colwell, "Microbial degradation of hydrocarbons in the environment.," Microbiol. Rev., vol. 54, no. 3, pp. 305-315, 1990.

[6] B. Klein, V. Grossi, P. Bouriat, P. Goulas, and R. Grimaud, "Cytoplasmic wax ester accumulation during biofilm-driven substrate assimilation at the alkane-water interface by Marinobacter hydrocarbonoclasticus SP17," Res. Microbiol., vol. 159, no. 2, pp. 137144, 2008.

[7] M. Salta, J. A. Wharton, S. P. Dennington, P. Stoodley, and K. R. Stokes, "Anti-biofilm performance of three natural products against initial bacterial attachment," Int. J. Mol. Sci., vol. 14, no. 11, pp. 21757 21780, 2013.

[8] M. T. Meyer, V. Roy, W. E. Bentley, and R. Ghodssi, "Development and validation of a microfluidic reactor for biofilm monitoring via optical methods," J. Micromechanics Microengineering, vol. 21, no. 5, p. 54023, 2011.

[9] G. E. Kapellos, "MICROSCALE STUDY OF BIOFILM DYNAMICS AT THE OIL-WATER INTERFACE George E. Kapellos Department of Chemical Engineering, University of Patras, 26504 Patras, Greece," pp. 4-6, 2015.

[10] G. M. Whitesides, "The origins and the future of microfluidics," Nature, vol. 442, no. 7101, pp. 368-373, Jul. 2006.

[11] S. Guo, C. A. Stevens, T. D. R. Vance, L. L. C. Olijve, L. A. Graham, R. L. Campbell, S. R. Yazdi, C. Escobedo, M. Bar-Dolev, V. Yashunsky, I. Braslavsky, D. N. Langelaan, S. P. Smith, J. S. Allingham, I. K. Voets, and P. L. Davies, "Structure of a 1.5-MDa adhesin that binds its Antarctic bacterium to diatoms and ice.," Sci. Adv., vol. 3, no. 8, p. e1701440, 2017.

[12] A. Tay, A. Pavesi, S. R. Yazdi, C. T. Lim, and M. E. Warkiani, "Advances in microfluidics in combating infectious diseases," Biotechnology Advances. 2016.

[13] S. Rismani Yazdi, R. Nosrati, C. A. Stevens, D. Vogel, P. L. Davies, and C. Escobedo, "Magnetotaxis Enables Magnetotactic Bacteria to Navigate in Flow," Small, vol. 1702982, p. 1702982, Dec. 2017.

[14] S. R. Yazdi, A. Shadmani, A. Hierlemann, and O. Frey, "Microfluidic hanging-drop platform for parallel closed-loop multi-tissue experiments," in 2015 28th IEEE International Conference on Micro Electro Mechanical Systems (MEMS), 2015, vol. 2015-Febru, no. February, pp. 535-538. 
[15] M. Zimmermann, P. Hunziker, and E. Delamarche, "Valves for autonomous capillary systems," Microfluid. Nanofluidics, vol. 5, no. 3, pp. 395-402, 2008.

[16] J. Melin, N. Roxhed, G. Gimenez, P. Griss, W. Vanderwijngaart, and G. Stemme, "A liquid-triggered liquid microvalve for on-chip flow control," Sensors Actuators B Chem., vol. 100, no. 3, pp. 463-468, 2004.

[17] M. I. Mohammed, E. Abraham, and M. P. Y. Desmulliez, "Rapid laser prototyping of valves for microfluidic autonomous systems," $J$. Micromechanics Microengineering, vol. 23, no. 3, p. 35034, 2013.

[18] T. Wu and Y. Suzuki, "Design, microfabrication and evaluation of robust high-performance superlyophobic surfaces," Sensors Actuators, $B$ Chem., vol. 156, no. 1, pp. 401-409, 2011.

[19] S. Rismani Yazdi, A. Shadmani, S. C. Bürgel, P. M. Misun, A. Hierlemann, and O. Frey, "Adding the 'heart' to hanging drop networks for microphysiological multi-tissue experiments," Lab Chip, vol. 15, no. 21, pp. 4138-4147, 2015.

[20] C. a Schneider, W. S. Rasband, and K. W. Eliceiri, "NIH Image to ImageJ: 25 years of image analysis.," Nat. Methods, vol. 9, no. 7, pp. 671-5, Jul. 2012. 